\title{
Characterization of a hybrid ion trap
}

\author{
C Richard Arkin, Brian Goolsby, D.A. Laude* \\ Department of Chemistry and Biochemistry, The University of Texas at Austin, Austin, TX 78712, USA
}

Received 8 April 1998; accepted 23 October 1998

\begin{abstract}
A new ion trap is constructed of a cylindrical ring electrode and hyperbolic end caps. The premise is to determine the effect ring electrode geometry has on the operation of the ion trap. A model for the potential and electric field within the trap is developed. The model is used to show how adjusting the geometric parameters of the cell could be used to reduce the presence of higher order fields or optimize other desired properties. The stability diagram is mapped experimentally and using the standard definition for $q_{z}$, the $q_{\text {eject }}$ was determined to be 0.76 . A proposed modification for the definition of $q_{z}$, which adjusts for the shape of the cylindrical ring electrode, results in a $q_{\text {eject }}$ of 0.93 . The trap is shown to have a linear scanning relationship, resolved isolation and unit mass resolution. (Int J Mass Spectrom 190/191 (1999) 47-57) @ 1999 Elsevier Science B.V.
\end{abstract}

Keywords: Ion trap mass spectrometry; Ion detection; Ion trap design; Mass spectrometry instrumentation; Trapped ion cell

\section{Introduction}

There have been a variety of radio-frequency ion traps that do not conform to the standard hyperbolic geometry described in the literature [1-11]. There are several reasons to deviate from the standard geometry, such as increased ion storage [1], rapid ion extraction [2], prolonged ion storage [3], miniaturization [4], and ease of machining [5]. We consider here a different geometry with which to study the effect of ring electrode geometry on the performance of ion trap operation. The justification is that ion trajectory in mass selected instability mode is primarily along the $z$ axis, and specifically we show that the ring electrode geometry can be significantly modified with minimal detriment to performance. To test this hy-

* Corresponding author. E-mail: DALaude@mail.utexas.edu Dedicated to J.F.J. Todd and R.E. March in recognition of their original contributions to quadrupole ion trap mass spectrometry. pothesis, the standard hyperbolic ring electrode was replaced with a cylindrical ring electrode.

The cell under consideration is a hybrid between the standard hyperbolic ion trap and the cylindrical ion trap (CIT). Performance in the hyperbolic ion trap has been well characterized [12] and the CIT is also well understood [7-11]. This new hybrid trap is characterized first by the development of a model to describe the electric potential and electric field within the volume of the cell. Second, the stability diagram is experimentally determined and related to the $a_{z}$ and $q_{z}$ dimensionless parameters of a theoretical hyperbolic trap. Last, the mass scan and isolation capabilities are discussed.

\section{Theoretical}

Although numerical solutions to the potential distribution within an ion trap cell is common with the 


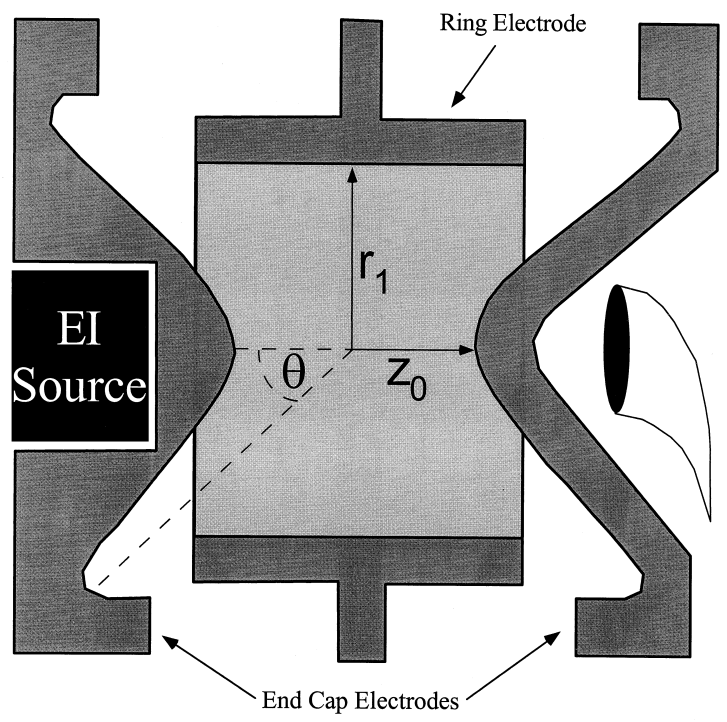

Fig. 1. Pictorial representation of the hybrid ion trap geometry used in the experiments with the definition of the relevant parameters.

software available today, the derivation of an analytical solution often provides insight unavailable through numerical analysis or simulation. For this purpose a solution to the electric potential and electric field within the hybrid trap is derived.

\subsection{Electric potential}

The electric potential produced within the volume of the trap is developed where a potential is applied to the ring electrode and the end caps are grounded (applying the potential in this manner is designated as mode II of operation [13]). The trap consists of two hyperbolic end cap electrodes and one cylindrical ring electrode which are defined by Eqs. (1) and (2), respectively, and illustrated in Fig. 1

$$
\begin{aligned}
& \frac{z^{2}}{z_{0}^{2}}-\frac{r^{2}}{z_{0}^{2} \tan ^{2} \theta}=1 \\
& r=r_{1}
\end{aligned}
$$

The parameters used to define the electrodes are the vertex and asymptotic angle of the end cap electrodes ( $z_{0}$ and $\theta$, respectively), the inner radius of the ring electrode $\left(r_{1}\right)$, the radial displacement $(r)$ and the axial displacement $(z)$.

A model to describe the electric potential and the electric field is developed in a method similar to March and co-workers [9]. The primary assumption here is that both the cylindrical ring electrode and the hyperbolic end cap electrodes extend toward infinity.

Using the method of separation of variables, the general solution to the Laplace equation with no angular dependence (3) is given in Eq. (4):

$$
\begin{aligned}
\nabla^{2} \Phi & =\nabla^{2} R(r) Z(z) \\
& =\left[\frac{\partial^{2}}{\partial r^{2}}+\frac{1}{r} \frac{\partial}{\partial r}+\frac{\partial^{2}}{\partial z^{2}}\right] R(r) Z(z) \\
& =0
\end{aligned}
$$

$\Phi(r, z)=R(r) Z(z)=A \cos (c z) I_{0}(c r)$

where $c$ is a constant resulting from the separation of variables, $A$ is the accumulation of integration constants, and $I_{0}(x)$ is the zero order modified Bessel function of the first kind [10].

In order to determine the exact solution for the model potential within the hybrid trap, the boundary conditions must be considered. For the ring electrode, the boundary condition is given in Eq. (5) where the potential at the electrode is the applied potential, $V_{0}$; $V_{\mathrm{dc}}$ is the direct current component of the applied potential, $V_{\text {rf }}$ is the amplitude of the oscillating component of potential applied, $\Omega$ is the frequency of the oscillating potential and $t$ is time.

$\Phi\left(r_{1}, z\right)=V_{0}=V_{\mathrm{dc}}+V_{\mathrm{rf}} \cos (\Omega t)$

For the hyperbolic end caps, which are at ground potential, the boundary condition is represented by Eq. (6), where $\rho$ is defined by Eq. (7)

$$
\begin{aligned}
& \Phi\left(r, \pm z_{0} \rho\right)=0 \\
& \rho \equiv \sqrt{1+\left(r^{2} / z_{0}^{2} \tan ^{2} \theta\right)}
\end{aligned}
$$

Being dependent on two variables, a hyperbolic boundary condition presents a difficulty; therefore, a coordinate transformation is used which will convert the hybrid cell into a cylindrical cell, by a method 
similar to that used by $\mathrm{O}$ and Schuessler [6]. Using the transformations of Eqs. (8) and (9),

$u=r$

$\nu=\frac{z}{\rho}$

the general solution to the Laplace equation becomes

$\Phi(u, \nu)=A \cos (c \nu \rho) I_{0}(c u)$

Following coordinate transformation, the boundary conditions become

$\Phi\left(u, \pm z_{0}\right)=0$

$\Phi\left(r_{1}, \nu\right)=V_{0}$

for the end cap and ring electrodes, respectively. The end cap boundary condition results in $\cos \left(c z_{0}\right)=0$, giving

$c_{n}=\frac{2 n+1}{2 z_{0}} \pi$

The final boundary condition is used, in conjunction with Fourier's theorem, to determine the integration constant, $A_{n}$, which is

$A_{n}=\frac{(-1)^{n} 2 V_{0}}{c_{n} z_{0} I_{0}\left(c_{n} r_{1}\right)}$

Thus the solution of the potential in $(u, \nu)$ space is

$\Phi(u, \nu)=\frac{2 V_{0}}{z_{0}} \sum_{n=0}^{\infty} \frac{(-1)^{n}}{c_{n}}\left(\frac{I_{0}\left(c_{n} u\right)}{I_{0}\left(c_{n} r_{1}\right)}\right) \cos \left(c_{n} \nu\right)$

Using Eqs. (8) and (9) the potential within the hybrid ion trap, in cylindrical coordinates is

$\Phi(r, z)=\frac{2 V_{0}}{z_{0}} \sum_{n=0}^{\infty} \frac{(-1)^{n}}{c_{n}}\left(\frac{I_{0}\left(c_{n} r\right)}{I_{0}\left(c_{n} r_{1}\right)}\right) \cos \left(\frac{c_{n} z}{\rho}\right)$

The isopotentials resulting from Eq. (16) are shown in Fig. 2(a).

\subsection{Electric field}

The electric field within the hybrid trap can be found by taking the gradient of Eq. (16)

$\mathbf{E}=\nabla \Phi=\frac{\partial \Phi}{\partial r} \mathbf{r}+\frac{1}{r} \frac{\partial \Phi}{\partial \theta} \theta+\frac{\partial \Phi}{\partial z} \mathbf{z}$

The scalar of the electric field for the hybrid trap is

$|\mathbf{E}|=\sqrt{\left(\frac{\partial \Phi}{\partial r}\right)^{2}+\left(\frac{\partial \Phi}{\partial z}\right)^{2}}$,

where

$$
\begin{gathered}
\frac{\partial \Phi}{\partial r}=\frac{2 V_{0}}{z_{0}} \sum_{n=0}^{\infty} \frac{(-1)^{n}}{I_{0}\left(c_{n} r_{1}\right)}\left[I_{1}\left(c_{n} r\right) \cos \left(\frac{c_{n} z}{\rho}\right)\right. \\
\left.-\frac{2 r}{\rho^{3} z_{0}^{2} \tan ^{2} \theta} I_{0}\left(c_{n} r\right) \sin \left(\frac{c_{n} z}{\rho}\right)\right], \\
\frac{\partial \Phi}{\partial z}=\frac{-2 V_{0}}{z_{0} \rho} \sum_{n=0}^{\infty}(-1)^{n}\left(\frac{I_{0}\left(c_{n} r\right)}{I_{0}\left(c_{n} r_{1}\right)}\right) \sin \left(\frac{c_{n} z}{\rho}\right)
\end{gathered}
$$

and $I_{1}(x)$ is the first order modified Bessel function of the first kind.

\subsection{Evaluation of potential and optimization of geometry}

Using the analytical approximations in Eqs. (16) and (18), the potential and field within the hybrid trap can be determined. The isopotentials resulting from Eq. (16) are shown in Fig. 2(a). Eqs. (16) and (18) can also be used to optimize the trap geometry.

The quality of a potential (or electric field) can be evaluated in terms of the extent of poles which contribute to the potential. Any potential distribution with cylindrical symmetry can be described by Eq. (21)

$\Phi(r, z)=\sum_{n=0}^{\infty} A_{n}\left(\sqrt{r^{2}+z^{2}}\right)^{n} P_{n}\left(\frac{z}{\sqrt{r^{2}+z^{2}}}\right)$

where $A_{n}$ are scaling factors for each pole order (e.g. $n=0-4$ corresponds to the monopole, dipole, qua- 

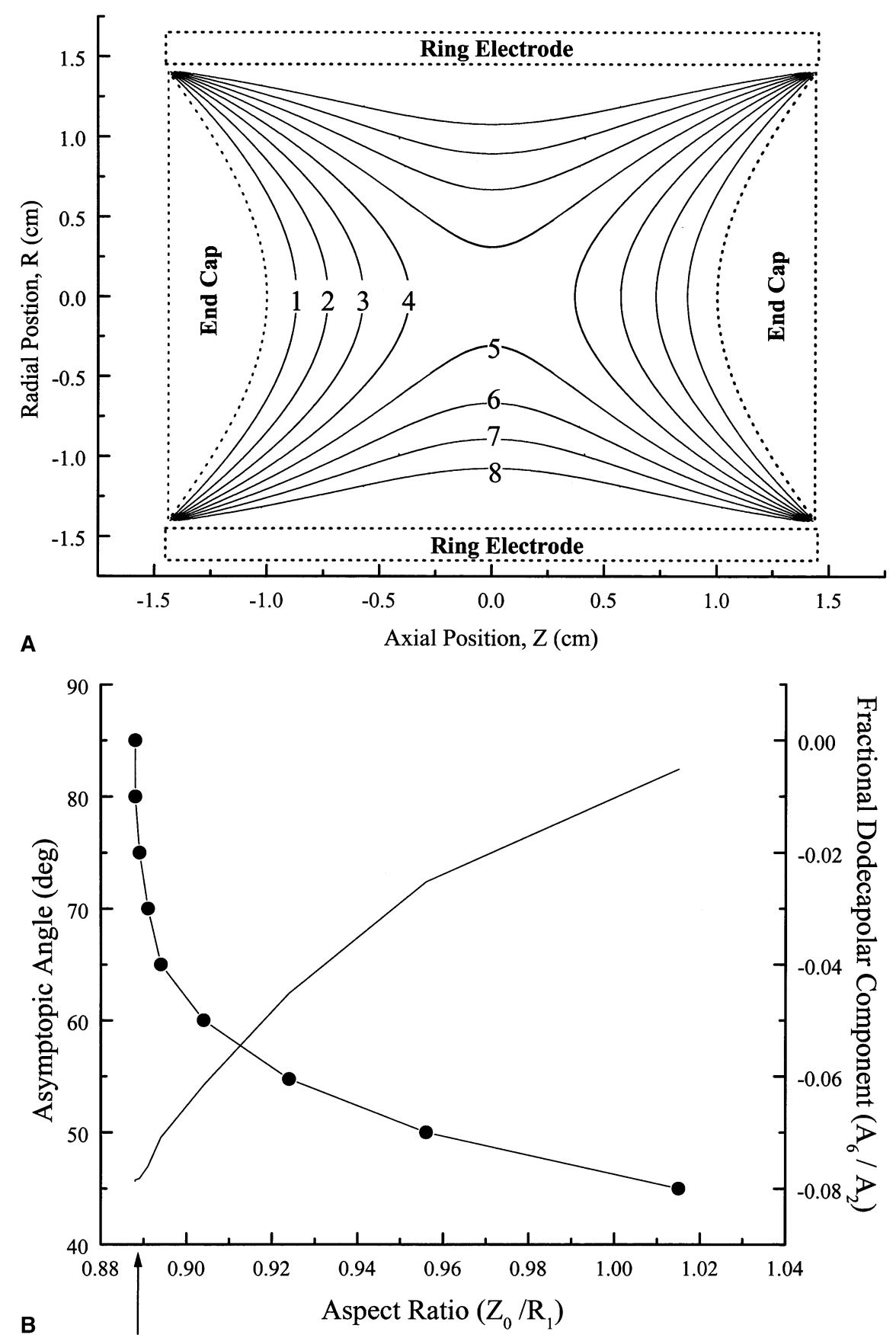

Fig. 2. (a) Isopotential plot of Eq. (16), with $r_{1}=1.4 \mathrm{~cm}, z_{0}=1.0 \mathrm{~cm}$, and $\theta=53.6^{\circ}$. The applied potential difference between the ring electrode and end cap electrodes is ten units. (b) Plot showing how adjustment of the asymptopic angle $(\theta)$ and the aspect ratio $\left(z_{0} / r_{1}\right)$ allows not only the octopolar component of the electric potential to be removed (closed circle), but that other parameters, such as the fractional dodecapolar component (dashed line), can be optimized. The arrow indicates the only aspect ratio which the CIT has a zero octopolar term, which also corresponds to the limit as $\theta$ approaches $90^{\circ}$ for the hybrid trap. (The CIT aspect ratio was calculated to be 0.888 , using the equations in [9].) 
drupole, hexapole and octopole, respectively), and $P_{n}(x)$ is the Legendre polynomial [15]. The $A_{n}$ terms can be determined through some simplifications; e.g. the $z$ axis of the electric field with mirror symmetry is represented by

$\left.\frac{\partial \Phi}{\partial z}\right|_{r=0}=\sum_{n=1}^{\infty}(-1)^{n}(2 n) A_{2 n} z^{2 n-1}$

In a perfect quadrupole ion trap, only the monopolar and quadrupolar terms ( $A_{0}$ and $A_{2}$, respectively) would be nonzero; however, in practice all real electrode arrangements have some higher order terms. In order to reduce the presence of higher order terms, the aspect ratio $\left(r_{1} / z_{0}\right)$ is optimized to produce the $A_{n}$ terms near zero $(n>2)$, as is done with the hyperbolic [16] and cylindrical ion traps [7].

Using Eq. (20), the $A_{n}$ terms for the hybrid trap are determined, and as expected an aspect ratio is found where the octopolar term $\left(A_{4}\right)$ is zero. However, unlike the CIT which has only one aspect ratio with $A_{4}=0$, the hybrid trap has the advantage of varying the asymptotic angle, $\theta$ [17]. This allows for an aspect ratio where $A_{4}=0$ for each angle. The benefit of this added degree of freedom is that the trap can be optimized for other variables. Some examples include the optimization of the dodecapolar term, pseudopotential well depth, or ion capacity without an octopolar term.

Fig. 2(b) shows the relationship between the aspect ratio and asymptopic angle where $A_{4}=0$. Fig. 2(b) also shows how a particular combination of aspect ratio and asymptopic angle may be chosen to optimize the dodecapolar term. For example, if one chose an asymptopic angle of $50^{\circ}$, an aspect ratio of 0.956 would produce a potential distribution with no octopolar $\left(A_{4}\right)$ component, as well as a fractional dodecapolar $\left(A_{6} / A_{2}\right)$ contribution of -0.025 .

\section{Experimental}

For these experiments a modified ITMS (Finnigan, San Jose, CA) mass spectrometer was used for the determination of the stability diagram. The ring elec- trode was replaced with a 304 stainless steel cylindrical electrode (i.d. $=37.85 \mathrm{~mm}$, width $=12.95 \mathrm{~mm}$, gap $=4 \mathrm{~mm})$. The end cap electrodes were not modified $\left(z_{0}=7.81 \mathrm{~mm}, \theta=54.2^{\circ}\right)$. The reagent, either dimethylether (DME), carbon tetrachloride or perfluorotributylamine (PFTBA), was used at a pressure of $4.0 \times 10^{-6}$ Torr, uncorrected. Ionization was achieved via electron ionization (EI) for period of 5.0 ms, and no bath gas was used.

Instrument control was performed with software associated with the ITMS system. Data acquisition was performed using a LeCroy 9400 dual channel digital oscilloscope (LeCroy, Chestnut Ridge, NY) and was transferred to a PC via GPIB, using LabVIEW (National Instruments, Austin, TX) software. Pressure measurements were made using a Bayard-Alpert, hot cathode ionization gauge (MKS Instruments, Boulder, $\mathrm{CO}$ ). The PFTBA and $\mathrm{CCl}_{4}$ ware degassed through two freeze-pump-thaw cycles. The DME was not purified. Samples were introduced into the vacuum manifold using a Granville-Phillips (Granville-Phillips Co., Boulder, CO) precision leak valve. Each mass spectrum consists of ten scans averaged on the oscilloscope. Following transfer to PC, each mass spectrum was smoothed with a ten-point adjacent average [18]. This data manipulation was performed in order to reduce the noise associated with circumventing the data processing features on the ITMS circuit board.

Stored-waveform inverse Fourier transform (SWIFT) [19,20] waveforms were produced using LabVIEW software developed in-house. These 32k data point waveforms used a sampling rate of $5 \mathrm{MHz}$ and were transferred to a Tektronix (Tektronix, Wilsonville, OR) model AWG 2005 arbitrary waveform generator via GPIB interface and triggered by the ITMS software.

The dc potential generated by the ITMS was determined by direct measurement with a digital voltmeter for each dc offset used. The rf potential generated was measured simultaneously with each part of the experimental scan from the 12-bit DAC output of the ITMS, using the second channel of the digital oscilloscope. A five-point calibration curve was produced between the 12-bit DAC output to the rf 
output test point of the ITMS, which is assumed to have a 1:100 ratio in amplitude when compared to the actual potential at the ring electrode.

\subsection{Determination of $\beta_{r}=0$ and $\beta_{z}=0$}

The experimental timing diagram (scan function) in determining the $\beta_{r}$ and $\beta_{z}$ boundaries is illustrated in Fig. 3(a). During the trapping event of the experiment, the trapping $\mathrm{rf}$ was set and dc potential was applied to the ring electrode. After a delay of $5.0 \mathrm{~ms}$, the $\mathrm{rf}$ potential was linearly ramped and the ejected ions were detected. A plot of ion abundance versus dc potential was generated and is illustrated in Fig. 3(b). As the $\beta$ line was approached, a sharp reduction in ion abundance was observed. This sharp portion of the curve was linearly extrapolated to determine the $x$ intercept. This $x$ intercept was defined as the dc potential for instability for the specific storage $\mathrm{rf}$ potential. The $\beta_{z}=0$ ejection line was determined with a positive dc offset and the $\beta_{r}=0$ ejection line was determined with a negative dc offset. This procedure was repeated for several rf storage potentials.

\subsection{Determination of $\beta_{z}=1$}

The $\beta_{z}=1$ line was determined simultaneously with the other lines. The points were determined by the dc and rf potentials at which the ion was detected via instability mode detection [21].

\subsection{Calculation of isobeta lines}

The isobeta lines, used to compare with the experimental data, were calculated from the $\beta=0$ and $\beta=1$ curves, described elsewhere [22]. Each polynomial was truncated at the eighth order of the polynomial.

\section{Results and discussion}

\subsection{Stability diagram}

Using the procedures outlined to determine the $\beta_{r}=0, \beta_{z}=0$ and $\beta_{z}=1$ lines, a stability dia- gram [Fig. 4(a)] was developed using the following equations [23] to calculate $a_{z}$ and $q_{z}$, respectively,

$a_{z}=\frac{-16 U_{\mathrm{dc}}}{(m / e)\left(r_{1}^{2}+2 z_{0}^{2}\right) \Omega^{2}}$

$q_{z}=\frac{8 V_{\mathrm{rf}}}{(m / e)\left(r_{1}^{2}+2 z_{0}^{2}\right) \Omega^{2}}$

where, $U_{\mathrm{dc}}$ is the dc potential applied to the ring electrode, $V_{\mathrm{rf}}$ is the rf potential amplitude applied to the ring electrode, $r_{1}$ is the inner radius of the ring electrode $(18.92 \mathrm{~mm}), z_{0}$ is the vertex of the hyperbolic end caps $(7.81 \mathrm{~mm})$, and $\Omega$ is the angular frequency of the rf potential $\left(6.9 \times 10^{6} \mathrm{rad} \mathrm{s}^{-1}\right)$ and $(\mathrm{m} / \mathrm{e})$ is the mass-to-charge ratio of the ion.

The experimentally determined stability diagrams for both fragments of DME have the same general shape, yet appear condensed when compared to the theoretical diagram for a hyperbolic trap. The reason for the reduced diagrams is related to the equations used to define $a_{z}$ and $q_{z}$. If one considers the effect a cylinder has on the potential at a fixed point when compared to a hyperboloid, the cylinder will produce a larger potential. In order for the potential to be the same at this fixed point, the hyperbola would need to be closer to the point. Since Eqs. (23) and (24) are for a hyperbolic ring electrode, there should be a scaling factor $(k<1)$ introduced into these equations which accounts for this effect. When the scaling factor is introduced to correct the radial parameter, the stability diagram approximates the theoretical hyperbolic stability diagram quite well. This is demonstrated in Fig. 4(b) in which Eqs. (25) and (26) are used to calculate $a_{z}$ and $q_{z}$, respectively, and with a value of $k=0.74$.

$a_{z}=\frac{-16 U_{\mathrm{dc}}}{(m / e)\left(k r_{1}^{2}+2 z_{0}^{2}\right) \Omega^{2}}$

$q_{z}=\frac{8 V_{\mathrm{rf}}}{(m / e)\left(k r_{1}^{2}+2 z_{0}^{2}\right) \Omega^{2}}$

The scaling factor of $k=0.74$ is inaccurate in two primary respects: the determination of the rf amplitude used an approximation to relate true amplitude at the cell to a test point on the rf generator circuit board 

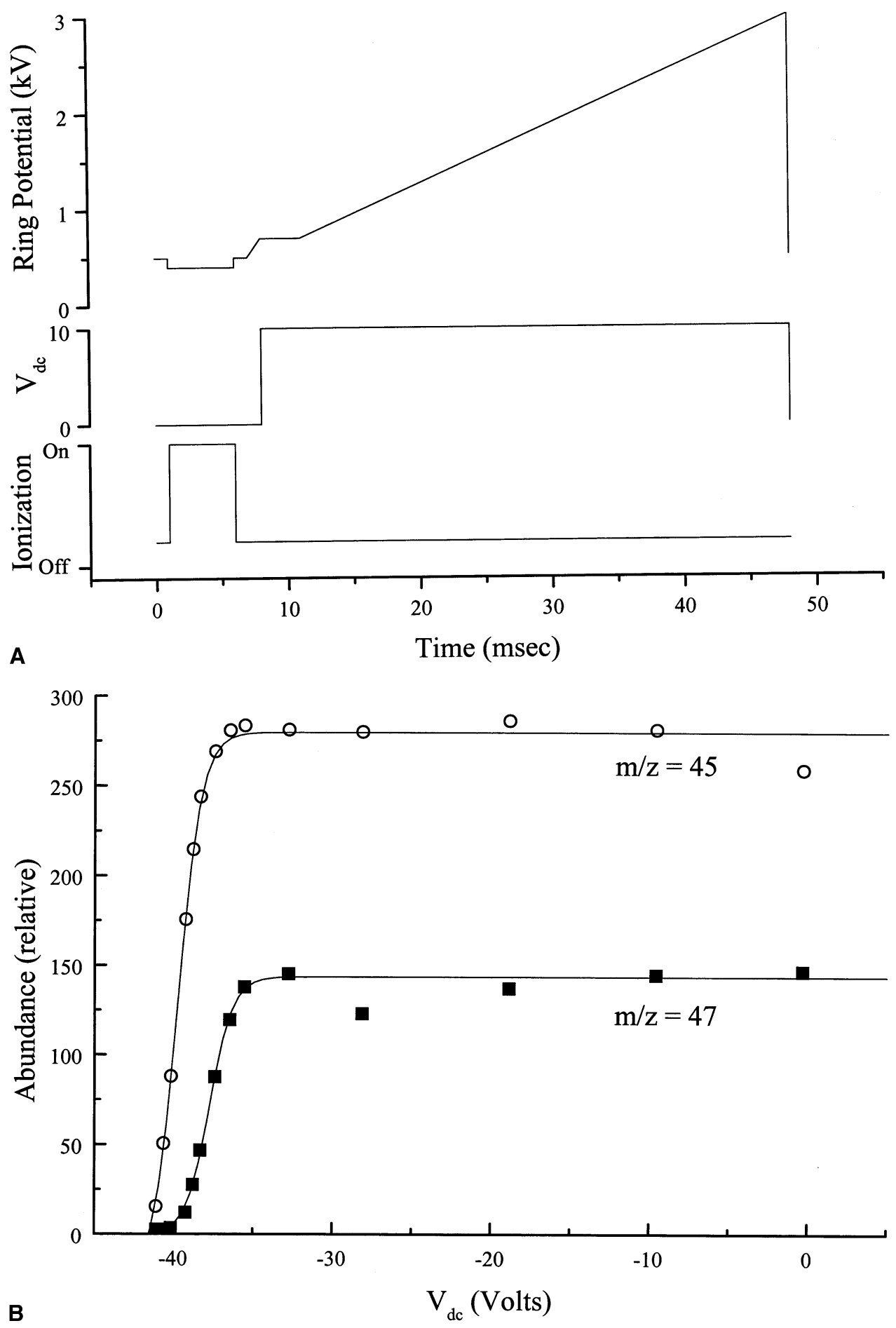

Fig. 3. Determination of the $\beta_{r}=0$ and $\beta_{z}=0$ isobeta lines for the hybrid trap. (a) Timing diagram for experiment used to determine the stability diagram. (b) Plot of ion abundance as the dc potential is decreased, at a constant storage trapping rf potential of $570 V_{\text {op}}$, showing decay in ion signal as the $\beta_{r}=0$ stability boundary is approached. 


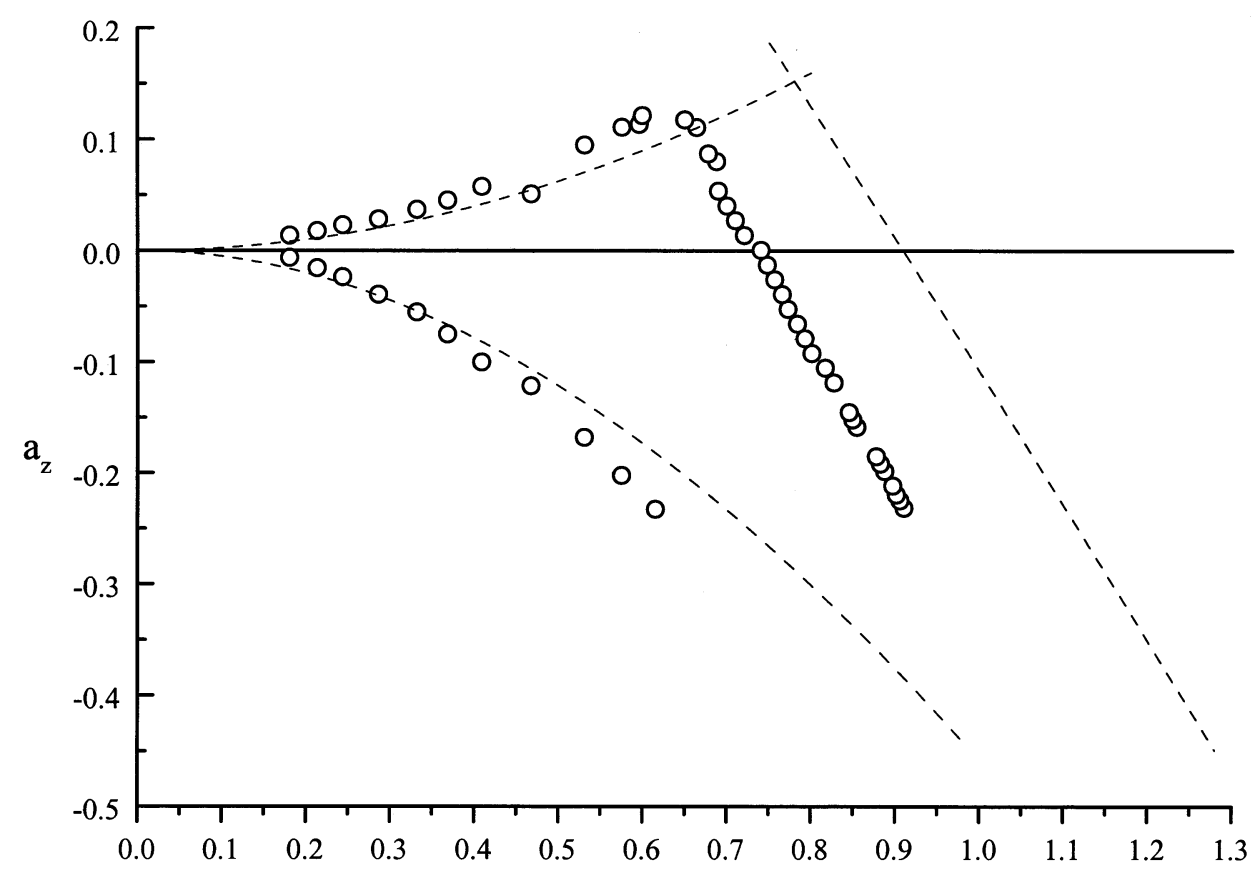

A

$\mathrm{q}_{\mathrm{z}}$

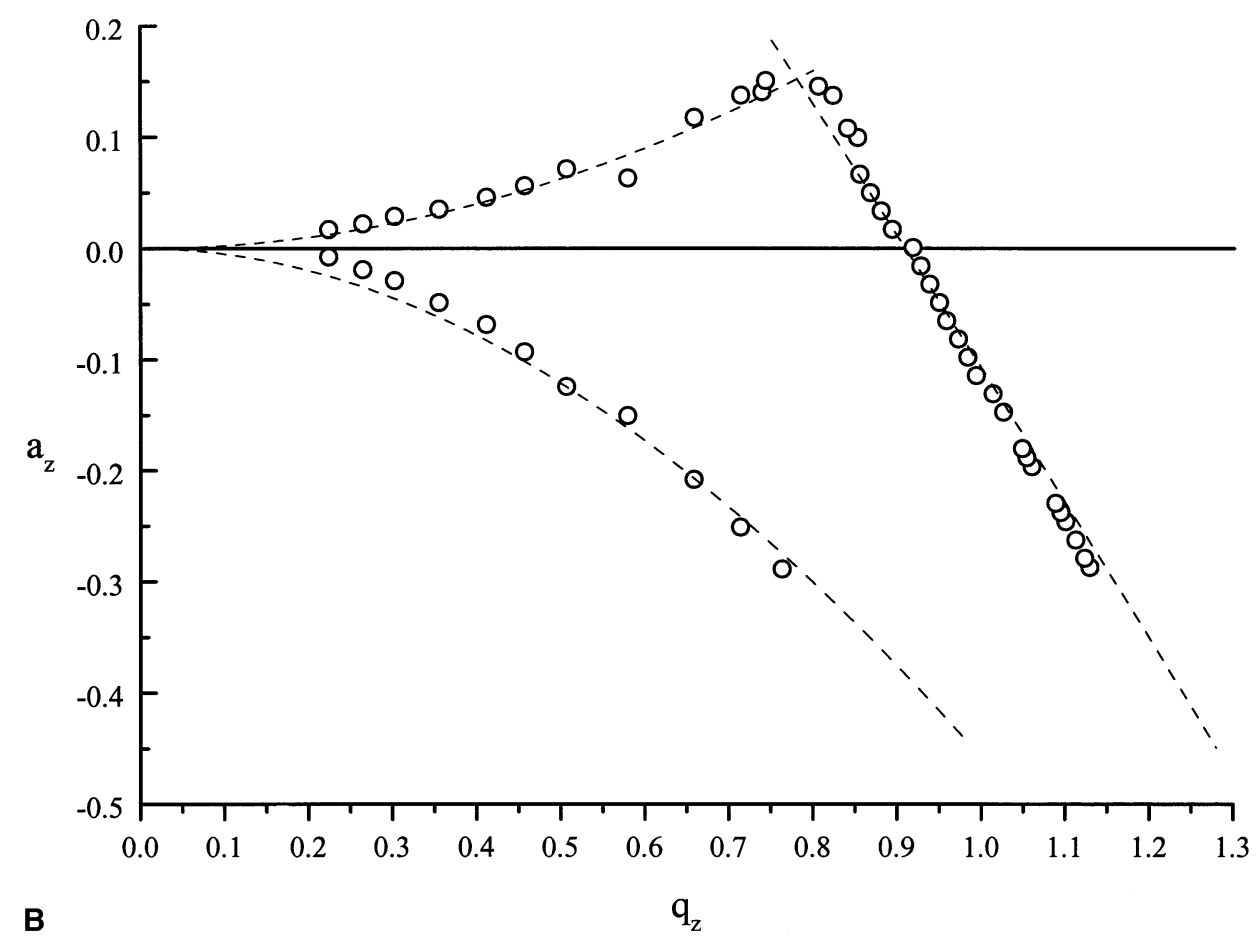

Fig. 4. Experimentally determined stability diagram (a) $\mathrm{m} / z=47$ DME fragment using the standard definitions of $a_{z}$ and $q_{z}$. (b) $m / z=47$ DME fragment using the modified definitions of $a_{z}$ and $q_{z}$, where $k=0.74$. (The stability diagrams for the $m / z=45$ DME fragment were essentially identical.) 

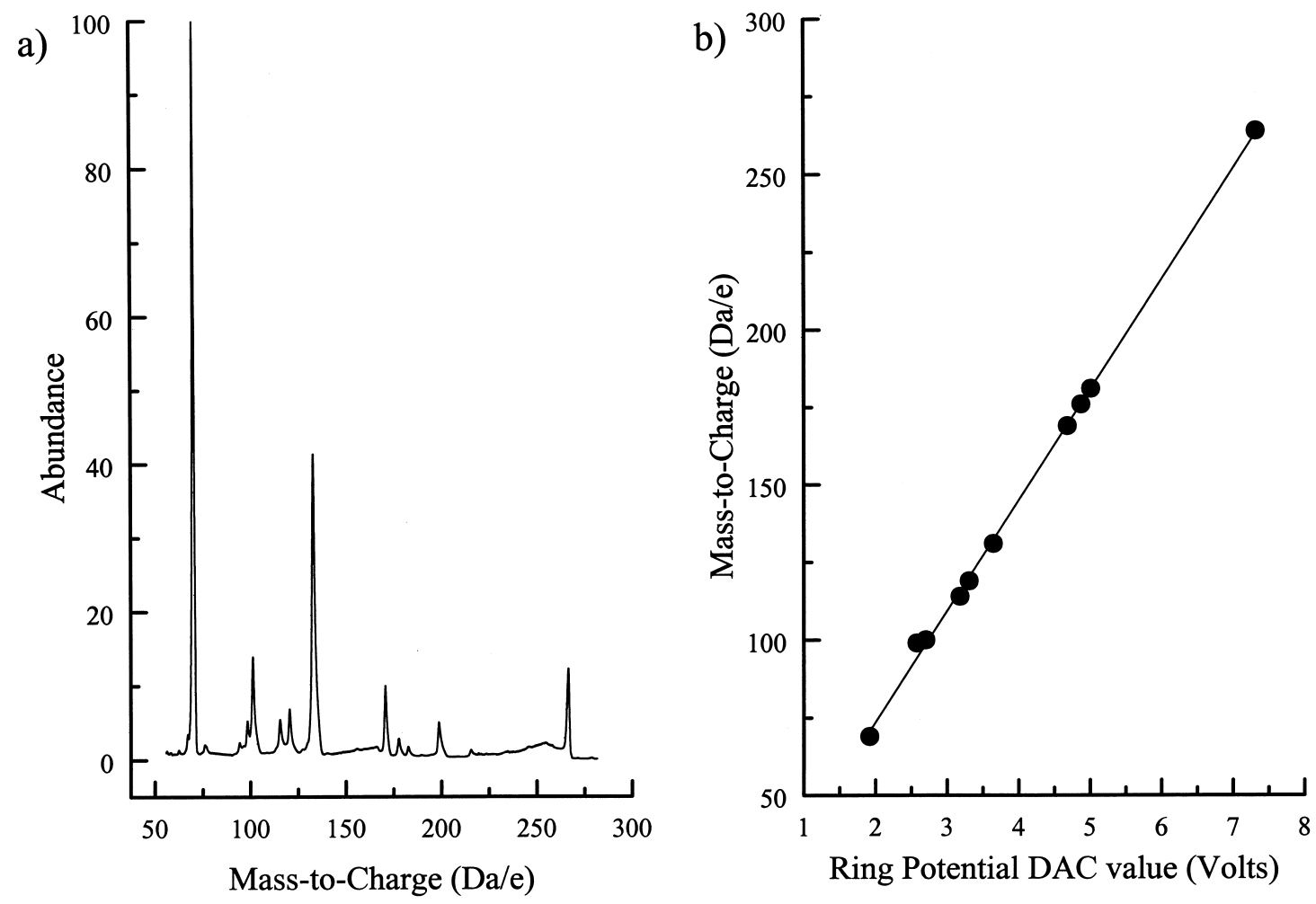

Fig. 5. (a) Mass spectrum of PFTBA. (b) Linearity of the applied rf potential, in terms of the D/A converter value, versus the mass-to-charge of the PFTBA fragment ion.

and the adjusted experimental stability diagram [Fig. 4(b)] does not correspond to the boundaries of the theoretical stability diagram. However, the fact that the experimental boundaries have a similar shape and nearly the same values as the theoretical boundaries, suggests that one could use a proportionality factor of 0.74 with equations previously developed [12] for the theoretical hyperbolic ion trap to estimate a number of desired variables such as secular ion frequency or pseudopotential well depth.

Although not evaluated in this study it is important to point out that evaluating the interior of the stability diagram can be as important as the determination of the stability boundaries. Since no real ion trap can produce a perfect quadrupolar potential, some higher order potentials will be present. The existence of these higher order potentials gives rise to a variety of nonlinear effects [24]. The superposition of higher order potentials on the quadrupolar potential can result in ion ejection when an ion resides on certain iso- $\beta$ lines, such as $\beta_{z}=2 / 3$ for hexapole and $\beta_{z}=$ $1 / 2$ for octopole. Such iso- $\beta$ lines which result in ion ejection are commonly called black canyons, and have been experimentally observed [25,26]. Black canyons generally reduce the retention of daughter ions in MS/MS and $\mathrm{MS}^{n}$ experiments and the efficient trapping of externally generated ions.

Higher order potentials are present in the hybrid trap, and one would predict that not only do black canyons exist for this geometry, but that their effect should be greater than for a hyperbolic geometry. However, through the choice of appropriate geometric parameters, such as aspect ratio and asymptopic angle, and experimental parameters, such as trapping potential, one could reduce the effects of these nonlinear resonances. 


\subsection{Mass scan}

A sample of PFTBA was used to determine the linearity of the mass scale relative to the rf potential. Fig. 5 shows the resulting PFTBA spectrum and the linear relationship between the rf potential applied to the ring electrode and the mass-to-charge ejected as predicted by both Eqs. (24) and (26). Using Eq. (24) the average $q_{\text {eject }}=0.76$. With a $k$ value of 0.74 , the average $q_{\text {eject }}$ is 0.93 using Eq. (26).

As the PFTBA spectrum indicates, the mass range is only $350 \mathrm{Da}$. This is a result of the large ring electrode radius $\left(r_{1}=18.92 \mathrm{~mm}\right)$ chosen [27] for this experiment and is not a result of the geometry.

\subsection{Resolution and isolation}

Two important practical features for a quadrupole ion trap are the ability to resolve peaks of close $\mathrm{m} / \mathrm{z}$ and the ability to isolate one for further ion manipulation. A sample of carbon tetrachloride, which produces three significant ion peaks with nominal $\mathrm{m} / \mathrm{z}$ of 117,119 and $121 \mathrm{Da} / e$, is used to demonstrate these properties in the hybrid trap. Fig. 6(a) shows, with the addition of a $440 \mathrm{kHz}$ dipolar signal across the end cap electrodes, that not only are the chlorine isotope peaks baseline resolved, but that the average resolution is 390 (FWHM). Using the SWIFT isolation technique the isolation capabilities of the hybrid trap are demonstrated in Fig. 6(b). Both the 117 and 121 peaks are absent and the retention of the 119 ions is $83 \%$, in abundance when compared to the Fig. 6(a).

\section{Conclusion}

The hybrid ion trap is capable of performing some of the same tasks as the standard hyperbolic ion trap. A model has been developed to describe the electric potential and field within the ion trap cell. The upper portion of the stability diagram was experimentally determined and shown to have the same general shape as that for the hyperbolic geometry. When a proportionality factor, $k$, was added to the equations defining the $a_{z}$ and $q_{z}$ values, the stability diagram approxi-
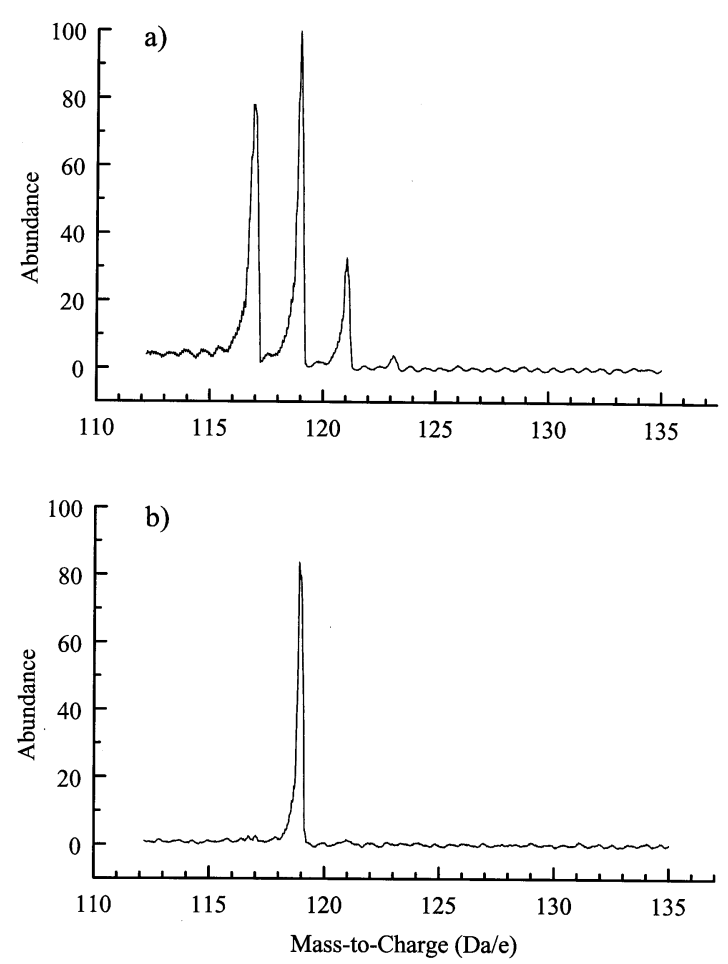

Fig. 6. (a) Resolution of the chlorine-35/chlorine-37 isotopes of the $\mathrm{CCl}_{3}^{+}$ion with axial modulation at $440 \mathrm{kHz}$ and $5.9 \mathrm{~V}_{\mathrm{op}}$. The 117 , 119 , and 121 nominal $\mathrm{m} / \mathrm{z}$ ions have a resolution (FWHM) of 343 , 408 , and 418, respectively. (b) SWIFT isolation of the $m / z=119$ ion, under the same conditions as in (a) except with the addition of a broadband SWIFT waveform of 180 to $300 \mathrm{kHz}$ with a single notch at $225 \pm 5 \mathrm{kHz}, 5 \mathrm{~V}_{\mathrm{op}}$

mated that of the hyperbolic model. This proportionality factor could be applied to estimate important parameters, such as secular ion frequency or pseudopotential well depth. A linear mass scale relative to applied rf potential was demonstrated. With the addition of a supplementary dipolar signal across the end cap electrodes, the useful technique of SWIFT isolation was shown with greater than $80 \%$ efficiency and unit mass resolution was demonstrated. Our conclusion is that modification of the ring electrode geometry affects the $a_{z}$ and $q_{z}$ parameters describing the ion stability boundary, yet has no apparent effect on the basic properties of the ion trap of linear mass scan, effective isolation and unit mass resolution. Currently the hybrid ion trap is being studied as a candidate for in situ image current detection [28,29]. Future work 
includes the optimization of the cell geometry for increased ion capacity.

\section{Acknowledgements}

CR.A. would like to thank Dr. Jennifer Brodbelt for use of the ITMS and Grady Rollins for the quality machine work. This work was supported by the NIH and The Welch Foundation.

\section{References}

[1] J.D. Prestage, G.J. Dick, L. Maleki, J. Appl. Phys. 66 (1989) 1013.

[2] Q. Ji, M.R. Davenport, C.G. Enke, J.F. Holland, J. Am. Soc. Mass Spectrom. 7 (1996) 1009.

[3] D.A. Church, J. Appl. Phys. 40 (1969) 3127.

[4] S.R. Jefferts, C. Monroe, E.W. Bell, D.J. Wineland, Phys. Rev. A 51 (1995) 3112.

[5] E.C. Beaty, J. Appl. Phys. 61 (1987) 2118.

[6] O. Chun-Sing, H.A. Schuessler, Int. J. Mass Spectrom. Ion Phys. 35 (1980) 305.

[7] J.M. Wells, E.R. Badman, R.G. Cooks, Anal. Chem. 70 (1998) 438.

[8] R.E. Mather, R.M. Waldren, J.F.J. Todd, Int. J. Mass Spectrom. Ion Phys. 33 (1980) 201.

[9] R.F. Bonner, J.E. Fulford, R.E. March, G.F. Hamilton, Int. J. Mass Spectrom. Ion Phys. 24 (1977) 255.

[10] M.-N. Benilan, C. Audoin, Int. J. Mass Spectrom. Ion Phys. 11 (1973) 421.

[11] D.B. Langmuir, R.V. Langmuir, R.F. Wuerker, U.S. Patent No. 3,065,640 (27 November 1962).

[12] R.E. March, J.F.J. Todd (Eds.), Practical Aspects of Ion Trap Mass Spectrometry, Vols 1, 2, and 3, CRC, Boca Raton, FL, 1995.
[13] R.F. Bonner, Int. J. Mass Spectrom. Ion Phys. 23 (1977) 249.

[14] E. Kreyszig, Advanced Engineering Mathematics, Wiley, New York, 1993, p. 241.

[15] M. Abramowitz, I.A. Stegun (Eds.), Handbook of Mathematical Functions, Dover, New York, 1965, p. 332.

[16] J. Louris, J. Schwartz, G. Stafford, J. Syka, D. Taylor, Proceedings of the 40th ASMS Conference on Mass Spectrometry and Allied Topics, Washington, DC, 31 May-5 June, 1992, p. 1003.

[17] Y. Wang, J. Franzen, Int. J. Mass Spectrom Ion Processes 112 (1992) 167.

[18] The PFTBA spectrum shown in Fig. 5(a) was smoothed a second time with a ten point adjacent average to facilitate identification of the lower abundance peaks.

[19] R.K. Julian Jr., R.G. Cooks, Anal. Chem. 65 (1993) 1827.

[20] A.G. Marshall, T.L. Wang, T.L. Ricca, J. Am. Chem. Soc. 107 (1985) 7893.

[21] G.C. Stafford Jr., P.E. Kelley, J.E.P. Syka, W.E. Reynolds, J.F.J. Todd, Int. J. Mass Spectrom Ion Processes 60 (1984) 85.

[22] R.E. March, R.J. Hughes, J.F.J. Todd, Quadrupole Storage Mass Spectrometry, Wiley-Interscience, New York, 1989, p. 45 .

[23] R.D. Knight, Int. J. Mass Spectrom. Ion Phys. 51 (1983) 127.

[24] R.E. March, J.F.J. Todd (Eds.), Practical Aspects of Ion Trap Mass Spectrometry, Vol. 1, CRC, Boca Raton, FL, 1995, Chap. 3.

[25] K.L. Morand, S.A. Lammert, R.G. Cooks, Rapid Commun. Mass Spectrom. 5 (1991) 491.

[26] F. Guidugli, P. Traldi, A.M. Franklin, M.L. Langford, J. Murrell, J.F.J. Todd, Rapid Commun. Mass Spectrom. 6 (1992) 229.

[27] The inner radius of the ring electrode $(18.92 \mathrm{~mm})$ was only chosen since it corresponds to the Finnigan ITMS ring electrode with the hyperbolic surface removed.

[28] CR. Arkin, D.A. Laude, Proceedings of the 45th ASMS Conference on Mass Spectrometry and Allied Topics, Palm Springs, CA, 1-5 June, 1997, p. 123.

[29] M. Soni, V. Frankevich, M. Nappi, R.E. Santini, J.W. Amy, R.G. Cooks, Anal. Chem. 68 (1996) 3314. 